\title{
Diabetes as a potential risk for periodontitis: association studies
}

\author{
Robert J. Genco ${ }^{1}$ | Wenche S. Borgnakke ${ }^{2}$ \\ ${ }^{1}$ Departments of Oral Biology, and Microbiology and Immunology, Center for Microbiome Research, University at Buffalo, Buffalo, New York, USA \\ ${ }^{2}$ Department of Periodontics and Oral Medicine, School of Dentistry, University of Michigan, Ann Arbor, Michigan, USA \\ Correspondence \\ Wenche S. Borgnakke DDS MPH PhD, Department of Periodontics and Oral Medicine, School of Dentistry, University of Michigan, Ann Arbor, MI, USA. \\ Email:wsb@umich.edu
}

\section{1 | INTRODUCTION}

Periodontal disease and diabetes mellitus are two of the most common chronic diseases in humans and they are linked. The association of periodontal disease and diabetes has been termed a "two-way relationship," with diabetes increasing the risk for periodontal disease and periodontal disease adversely affecting glycemic control and increasing the severity of complications of diabetes. ${ }^{1}$

Here we will discuss the evidence for the role of diabetes in increasing the risk for periodontitis, whereas the role of periodontal disease adversely affecting diabetes is discussed elsewhere in this volume.

\section{2 | BURDEN OF PERIODONTITIS}

Periodontitis affects $42.2 \%$ of dentate adults in the USA $\geq 30$ years of age. ${ }^{2}$ Mild/moderate periodontitis accounts for $34.4 \%$ and severe periodontitis accounts for $7.8 \%$ of the total. Data from 37 countries show that severe periodontitis affects $11.2 \%$ of the population on average, ranging from $5 \%$ for individuals from Oceana to $20.4 \%$ among Latin Americans. It is the sixth most prevalent among 291 diseases assessed globally. ${ }^{3,4}$

\section{3 | BURDEN OF DIABETES MELLITUS}

Diabetes mellitus is a group of metabolic disorders characterized by hyperglycemia associated with defective insulin production, insulin action or both. In the USA, 30.3 million (9.4\%) adults had diabetes in 2017. ${ }^{5}$ Of these, 23.1 million were diagnosed and another 7.2 million were unaware of their diabetes. Prevalence has tripled worldwide from 2006 to $2017 .{ }^{6}$ Overall, type 2 diabetes accounts for $90 \%$ of the cases; type 1 or immune-mediated diabetes and gestational diabetes account for most of the rest. ${ }^{7}$
Other types of diabetes, including maturity-onset diabetes of the young and pancreatic disease or drug- and chemical-induced diabetes, are rare. ${ }^{8}$

The prevalence of diabetes varies among the countries of the world, with China, India and the USA having the highest prevalence. ${ }^{6}$ Prediabetes or increased risk for diabetes occurs in about one-third of US adults. It is defined as a fasting plasma glucose level of 100$125 \mathrm{mg} / \mathrm{dL}$ and/or elevated HbA1c of 5.7\%-6.4\%. Prediabetes predisposes to manifest diabetes, with about two-thirds converting in 3 years. ${ }^{5}$ Hyperglycemia affects one in six pregnancies worldwide, of which $86.4 \%$ are due to gestational diabetes. ${ }^{6} \mathrm{~A}$ large proportion of women with gestational diabetes will develop diabetes 3-6 years postpartum. ${ }^{9}$

\section{DIABETES COMPLICATIONS}

Hyperglycemia, especially of long duration, leads to diabetes complications and these complications are similar for all types of diabetes. Complications of diabetes include dehydration, hyperosmolar coma, poor wound healing and diseases such as myocardial infarction, stroke, limb ischemia, kidney failure, retinopathy leading to blindness, neuropathy, neurocognitive decline and foot infections, which can lead to amputation. ${ }^{8}$ Heart disease and stroke are the main causes of death among those with diabetes. Diabetic retinopathy is the leading cause of blindness; diabetes is the leading cause of kidney disease in the USA. Pregnant women with glycemia or gestational diabetes mellitus are at high risk of transgenerational effects on their offspring, including obesity, hypertension and kidney disease. ${ }^{6}$ Periodontal disease, which occurs in the majority of adults with diabetes, is also a complication of diabetes. The complications of uncontrolled diabetes are often devastating, resulting in increased risk for death, heart disease and stroke at rates two to four times higher than in individuals without diabetes. $^{10}$ 


\section{5 | DIABETES AND PERIODONTITIS}

\section{1 | Cross-sectional studies}

The most recent cross-sectional study defines the relationship of diabetes to periodontitis in over 10000 individuals representing over 143 million dentate adults in the USA examined from 2009 to $2012 .{ }^{11}$

These two 2-year NHANES cycles apply for the first time a full-mouth periodontal probing protocol, which enables the most accurate estimates of the prevalence of periodontitis. It should be noted that NHANES does not distinguish between the different types of diabetes.

From Figure 1 it can be seen that adults $\geq 30$ years of age with diabetes suffer from more moderate or severe periodontitis as compared with those with normal glucose levels at all age levels. ${ }^{11}$

Likewise, the prevalence of moderate/severe periodontitis is greater in those with poorly controlled diabetes and in those with controlled diabetes or prediabetes compared to participants without diabetes in both sexes (Fig. 2). Furthermore, that prevalence is greater in men compared to women within each of the three categories of glycemic control (results not shown).

Having no natural teeth (edentulism) is more common in those with diabetes and prediabetes in the age range of 45-64 years, but not among those 65 years or older. ${ }^{11}$

A well, there was more edentulism among those with elevated HbA1c levels as compared with those with good glycemic control. A similar finding was seen when the number of missing teeth was analyzed, ie more missing teeth in those with diabetes. Missing teeth were also greater in those with greater $\mathrm{HbA} 1 \mathrm{c}$.

From this study reprepresenting over 143 million adults in the USA, diabetes and hyperglycemia are associated with more severe periodontitis, edentulism, and tooth loss.

\section{6 | PERIODONTITIS IN TYPE 1 DIABETES}

The oral health status of 263 subjects with type 1 diabetes was compared with 208 with no diabetes (aged $11-18$ years). ${ }^{13}$ Almost $10 \%$

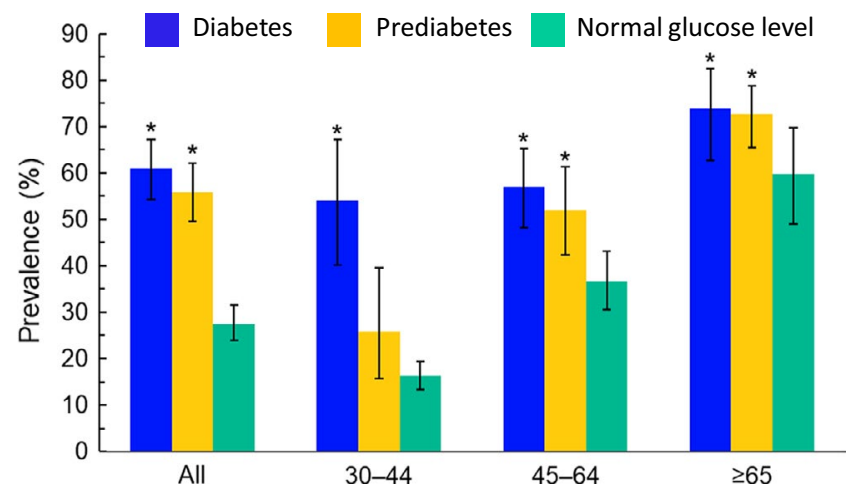

FIGURE 1 Prevalence of moderate/severe periodontitis ${ }^{12}$ among dentate adults aged $\geq 30$ years by diabetes status and age group - National Health and Nutrition Examination Survey (NHANES) 2009-2012. ${ }^{11}$ Error bars represent 95\% confidence intervals. ${ }^{*}$ p $<0.05$ compared to persons with normal glucose levels.

\section{Clinically important facts about the association of} diabetes and periodontal disease include:

- Type 1 and type 2 diabetes, as well as gestational diabetes, increase the risk for periodontal disease, especially if uncontrolled.

- Diabetes also increases the risk of tooth loss, which interferes with diet, often resulting in poor glycemic control.

- Diabetes can result in increased oral fungal infection and fissured tongue. Diabetes can also lead to burning mouth and alterations in taste sensation, both of which can interfere with eating, resulting in poor nutrition.

- Appropriate control of blood sugar and weight and adequate exercise can result in good diabetes control and the prevention and management of periodontal disease can result in good oral health. Both can be obtained in patients with diabetes to help ensure a healthier life for many patients.

(9.8\%) of those with type 1 diabetes had evidence of periodontitis versus $3 \%$ in children without diabetes. Figure 4 shows radiographs from a 16-year-old female with type 1 diabetes and severe periodontitis from this study. Severe alveolar bone loss especially affecting the molars and incisors can be seen in this patient.

A population-based longitudinal study showed that subjects with uncontrolled type 1 diabetes suffered from greater loss of clinical attachment over 5 years. ${ }^{14}$ Moreover, uncontrolled type 1 diabetes, as well as uncontrolled type 2 diabetes, was associated with greater tooth loss over the 5 -year follow-up period.

Similarly, participants with type 1 diabetes had a greater bleeding index as well as more periodontal attachment loss than those

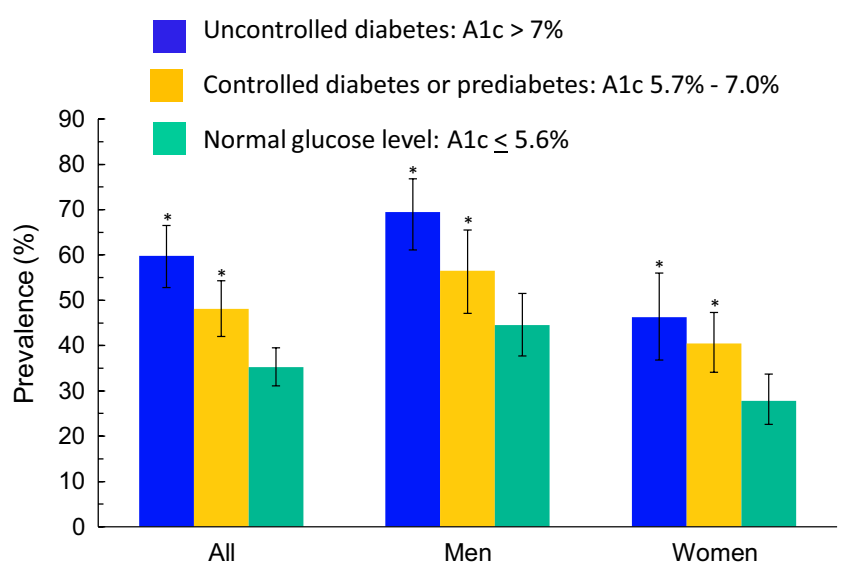

FIGURE 2 Prevalence of moderate/severe periodontitis ${ }^{12}$ among dentate adults aged $\geq 30$ years by diabetes (glycemic control) status and sex - National Health and Nutrition Examination Survey (NHANES) 2009-2012. ${ }^{11}$ A1c (HbA1c), glycated hemoglobin. Error bars represent $95 \%$ confidence intervals. ${ }^{*} p<0.05$ compared to persons with normal glucose levels. 


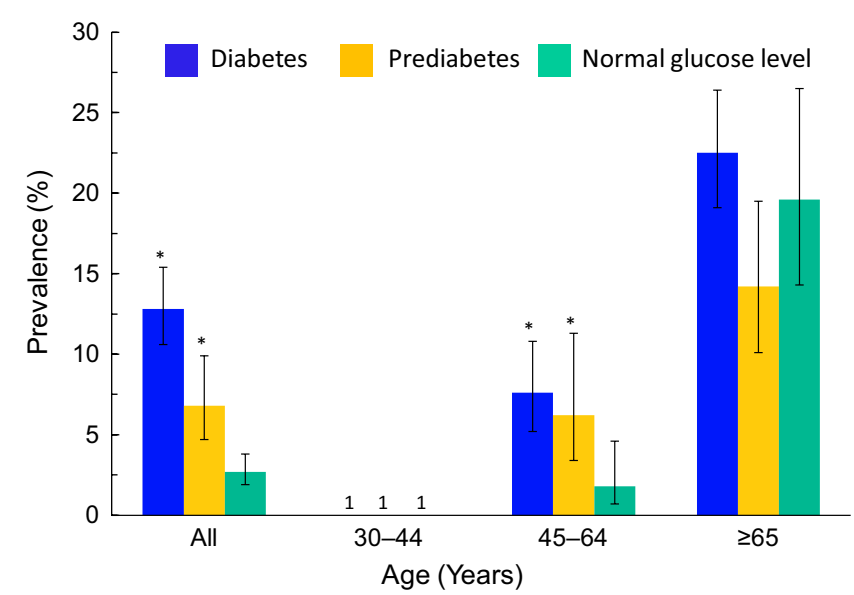

FIGURE 3 Prevalence of edentulism (missing all natural teeth) among adults aged $\geq 30$ years by diabetes status and age group - National Health and Nutrition Examination Survey (NHANES) 2009-2012. ${ }^{11}$

${ }^{1}$ Estimate is too unreliable to present; $£ 1$ case or relative standard error $>50 \%$. Error bars represent $95 \%$ confidence intervals. ${ }^{*} \mathrm{p}<0.05$ compared to persons with normal glucose levels.

with no diabetes, ${ }^{15}$ and those with deficient metabolic control and other diabetic complications had higher bleeding indices and greater periodontal pocket depths. The authors concluded that patients with type 1 diabetes show increased susceptibility to periodontal disease, especially those with poorer metabolic control or with diabetic complications.

\section{7 | GESTATIONAL DIABETES MELLITUS}

Analyses of NHANES III data showed that women with gestational diabetes had more severe periodontitis than pregnant women without gestational diabetes. ${ }^{16} \mathrm{~A}$ large prospective Indian study of primagravida demonstrated a strong association between gestational diabetes and periodontal disease with an adjusted hazard ratio of 2.85 (95\% Cl: $1.47-$ 5.53). Also, there was a more than 18 -fold increased risk for pre-eclampsia in those with both periodontal disease and gestational diabetes with an adjusted hazard ratio of 18.79 (95\% Cl: 7.45-47.40) compared to first-time pregnant women without any of the two diseases. ${ }^{16}$
It appears that individuals with most types of diabetes mellitus, including type 1 , type 2 , and gestational diabetes mellitus, especially if uncontrolled have more severe periodontitis than subjects with no diabetes.

\section{8 | LONGITUDINAL STUDIES}

From this long history of cross-sectional studies and case reports it is clear that patients with type 1 and type 2 diabetes, as well as gestational diabetes, suffer from more severe periodontal disease, after adjustment for common risk factors. As strong as the evidence for this association is, there still is a need to establish the temporal sequence of events: Does diabetes precede periodontitis and, hence, is possibly a risk factor, contributing to the pathogenesis of periodontitis? Alternatively, is periodontitis a condition that precedes or is concurrent with diabetes? There is a series of longitudinal studies that address these issues.

The earlier onset and greater severity of periodontal disease was documented in a longitudinal study of Pima Indians of the Gila River Native American Community. ${ }^{18}$ In this study, the incidence or number of new cases of periodontitis was 2.6 times greater in those with diabetes type 2 as compared with individuals with normal glucose levels when followed over 2-3 years. Figure $5 \mathrm{~A}-\mathrm{C}$ shows a subject who did not have diabetes at baseline, with little or no periodontal disease. However, she developed type 2 diabetes and severe periodontitis was seen on examination about 3 years later. ${ }^{18}$

This has been confirmed and extended in a population-based study in Germany. ${ }^{14}$ They studied a large population over 5 years and found that individuals with uncontrolled type 2 diabetes had greater progression of pocket depth and clinical attachment level compared with those with controlled or no diabetes.

Another longitudinal study followed patients for 5 years post-treatment during a periodontal maintenance regimen. ${ }^{19}$ The progression of periodontitis was significantly greater among patients with diabetes and poor glycemic control (mean $\mathrm{HbA1c} 9.1 \%$ ) as compared with those with diabetes and good control (mean HbA1c 6.1\%) and nondiabetics. The results from this study can be seen in Figure 6. Moreover, tooth loss was greater over the 5-year period in those with diabetes with poor glycemic control compared with those with good control or those with no diabetes.
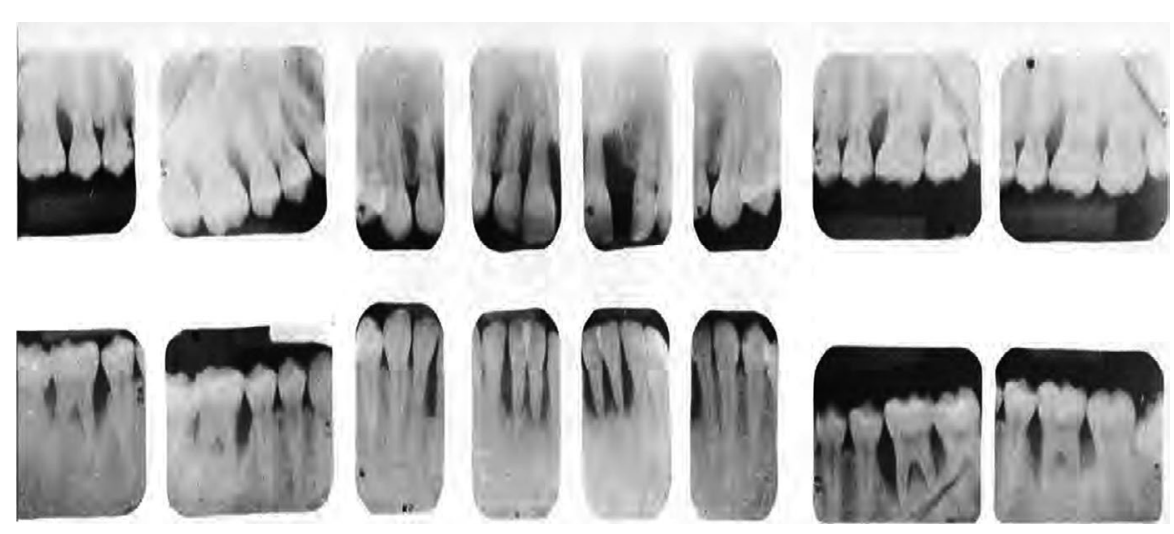

FIGURE 4 16-year-old, White female patient. Type 1 diabetes mellitus onset at age 14 years, duration 2 years. Note alveolar bone loss localized to first molars, especially mesial surfaces and central incisors. ${ }^{13}$ 

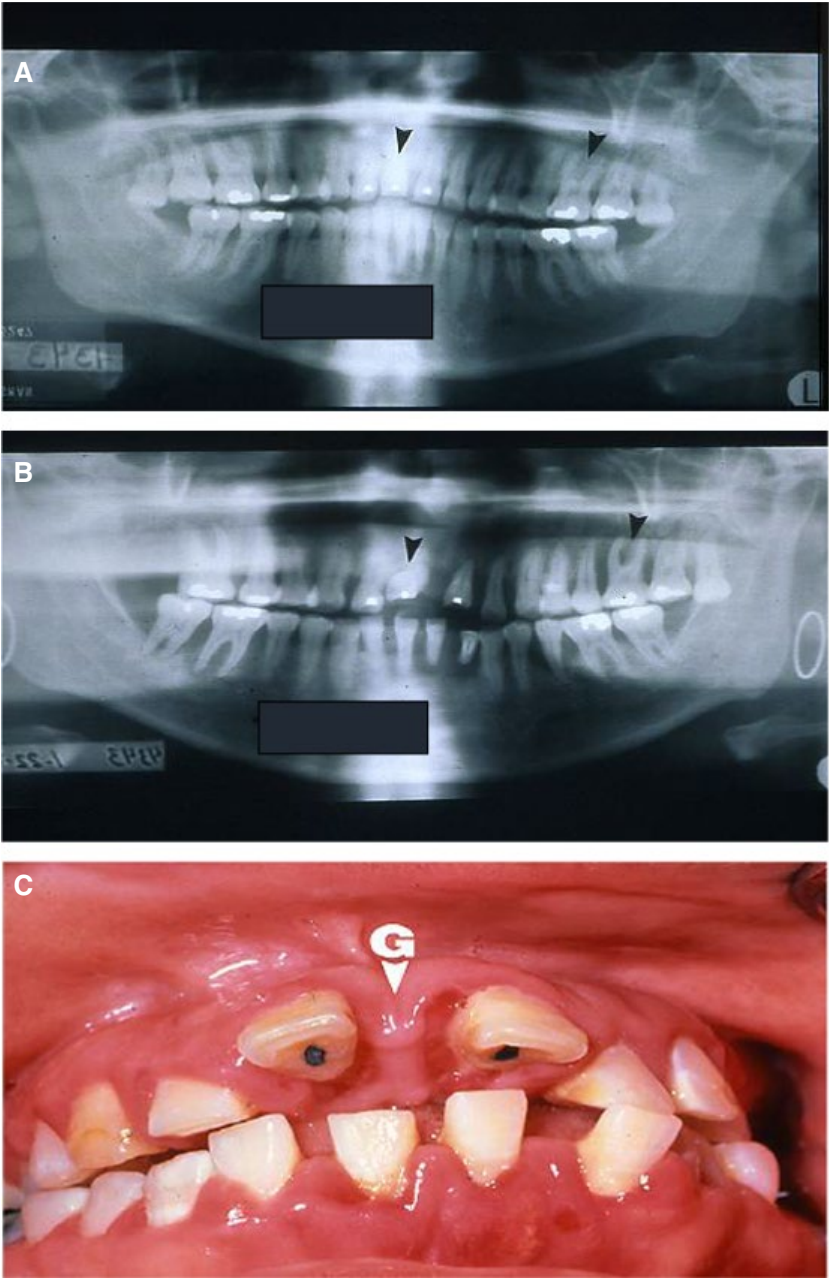

FIGURE 5 (A) 35-year old female, baseline examination, mild alveolar bone loss, no diabetes (radiograph). (B, C) Same patient 3 years later, now with severe periodontitis and uncontrolled type 2 diabetes [radiograph (B), clinical photograph (C)]. ${ }^{18}$

As described, there is greater edentulousness and partial tooth loss in individuals with diabetes based on cross-sectional studies. The longitudinal study by Demmer et al showed that greater tooth loss occurred in both type 1 and type 2 diabetes when uncontrolled, as compared with groups with controlled or no diabetes. ${ }^{14}$ Loss of teeth in individuals with diabetes is of great potential significance, as it often interferes with proper nutrition.

Based on the cross-sectional and longitudinal studies it is reasonable to conclude that diabetes mellitus is a risk factor for periodontitis and that diabetes precedes periodontitis, probably contributing to its pathogenesis. Evidence for mechanisms by which diabetes increases the risk for periodontal diseases in summarized elsewhere in this volume of Periodontology 2000.

\section{9 | COMMON RISK FACTORS}

Periodontal disease and diabetes share many risk factors, both modifiable and nonmodifiable. ${ }^{20-22}$ The modifiable risk factors common to diabetes and periodontal disease include smoking, excessive alcohol consumption, obesity, physical inactivity and excessive refined sugar consumption. It should be noted that periodontal disease is also a risk factor for worsening diabetes, and diabetes for more severe periodontitis.

The nonmodifiable risk factors common to diabetes and periodontal disease include higher age, male gender, minority race/ ethnicity, low socioeconomic status and genetic predisposition. ${ }^{21}$ It is clear then that in the management of periodontal disease, especially in patients with diabetes, strict attention to modifying smoking, excessive alcohol consumption, obesity, physical activity and high refined sugar consumption will benefit the management of both diseases. This can perhaps be best accomplished by a team approach, including a dentist and physician and their respective staff.

\section{0 | OTHER ORAL MANIFESTATIONS OF DIABETES}

Because diabetes and periodontitis practically share the same risk factors, both conditions will often occur in the same individuals. ${ }^{20-22}$ Moreover, several mechanisms involved in the metabolic syndrome called diabetes, such as hyperglycemia, insulin resistance, or glycose intolerance; dyslipidemia; various metabolic disturbances; cardiovascular dysfunction, such as endothelial dysfunction and hypertension; and reduced resistance to infection and delayed wound healing by inflammatory dysfunction, may result in various oral manifestations, symptoms of which subsequently further increase the risk of periodontitis. An extensive literature review supplemented with original population-based data specifically examines the periodontal complications of diabetes/hyperglycemia. ${ }^{23}$ Diabetes related oral conditions with the most common appearance deserve brief mention here and are further described in a comprehensive 2019 review by Verhulst and collaborators. ${ }^{24}$ The most important oral manifestation of diabetes in terms of both decrease in quality of life and impact on oral diseases that contribute to this decrease is probably dry mouth, which in turn can cause problems with speech, mastication, swallowing, and removable dentures, periodontitis and peri-implantitis, ${ }^{25}$ potentially caries, and eventually tooth loss. Moreover, candidiasis, fissured tongue, and other oral mucosal lesions are more prevalent in diabetes/hyperglycemia. Hyposalivation, burning mouth, and taste alterations can occur due to diabetic neuropathy. ${ }^{26}$ Importantly, medications the patients take for their diabetes and frequently occurring co-morbidities all have the potential to cause dry mouth, a strong risk factor for both periodontitis and caries. The two latter oral diseases are the major causes for tooth loss that in turn easily can lead to poor nutrition and further decrease the glycemic control due to the inability to bite off and chew healthy food items. This is due to the resulting need for a soft diet that often is laden with sugar, fat, and salt and thus prohibits patients with diabetes from following their dietary recommendations for optimal diabetes management. ${ }^{11}$ 

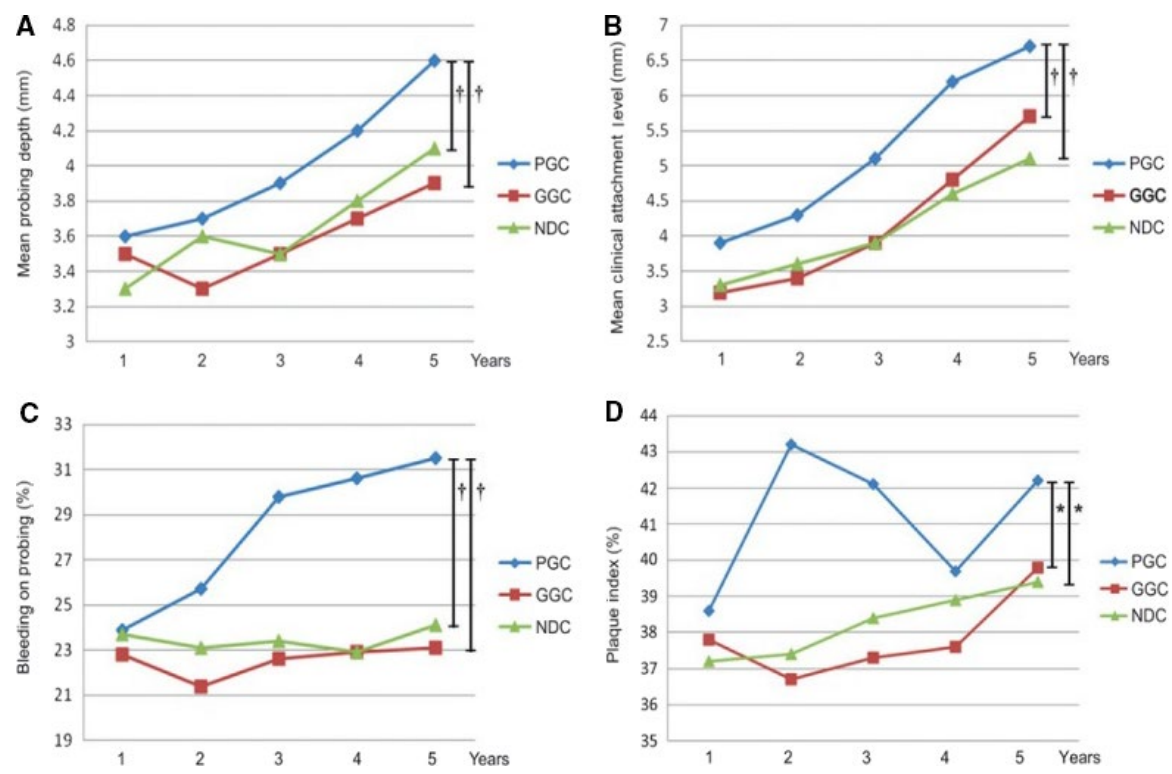

FIGURE 6 Mean in (A) periodontal probing depth (PPD), (B) clinical attachment loss (CAL), (C) bleeding on probing (BOP), and (D) plaque index (PI) during 5 years of periodontal maintenance therapy. At baseline: PGC GGC NDC ( $>>0.05)$, except for CAL: PGC > GGC NDC $(p<0.039)$. At final examination: PGC > GGC ( $p<0.001)$. PGC, poor glycemic control; GGC, good glycemic control; NDC, controls with no diabetes. *† PGC > NDC ( $<<0.01) ;{ }^{*} \dagger \mathrm{GGC} \sim \mathrm{NDC}(\mathrm{p}>0.05) .{ }^{*}$ Statistically significant increase in PI per year, using ANOVA and Bonferroni post hoc analysis $(\mathrm{p}<0.05)$. † Statistically significant increase in PPD, CAL, and BOP per year, using Welch test and Tamhane post hoc analysis $(p<0.05)$. Reprinted from Costa et $\mathrm{al}^{19}$ with permission.

\section{1 | IMPAIRED WOUND HEALING IN PATIENTS WITH DIABETES}

Delayed wound healing, including oral wounds and lesions, has been well known to occur in patients with diabetes. The factors involved in increasing oral disease include: hypoxia, dysfunction in fibroblasts and epidermal cells, impaired angiogenesis and neovascularization, high levels of metalloproteases, damage from reactive oxygen species and advanced glycation end products, neuropathy and multiple levels of decreased host immune resistance. ${ }^{27}$ Most of these factors are accentuated in uncontrolled diabetes and they adversely affect dental procedures that require wound healing.

Dental management of patients with diabetes requires special attention to their treatment planning and management, especially post-treatment. Proactive coordination of care with their physician to help ensure proper glycemic control is critical to long-term successful periodontal and implant therapy. Patients should be assessed regularly for periodontitis, and in those periodontally healthy, strict preventive measures instituted to prevent periodontal disease. If the patient with diabetes has periodontal disease, it should be treated definitively, reducing all periodontal pockets, establish glycemic control and control of other complications managed in coordination with the patient's physician. Good glycemic control and modification of common risk factors, such as smoking and obesity, as well as complete periodontal therapy and regular periodontal maintenance will help the patient achieve a lifetime of good oral as well as general health.

\section{SUMMARY}

Periodontitis and diabetes mellitus should be of great interest to clinicians because both are common diseases with more than 4 in 10 dentate adults suffering from periodontitis and about 1 in 10 adults having diabetes and because these two diseases are associated. This can have implications for the treatment plan and for the expected outcomes thereof. Importantly, the dental team may be the first to suspect undiagnosed diabetes based on the oral health status, first and foremost related to the periodontium.

\section{REFERENCES}

1. Grossi S, Genco RJ. Periodontal disease and diabetes mellitus: a two-way relationship. Ann Periodontol. 1998;3(1):51-61.

2. Eke PI, Thornton-Evans GO, Wei L, et al. Periodontitis in US adults: National Health and Nutrition Examination Survey 2009-2014. J Am Dent Assoc. 2018;149(7):576-588; 588.e1-588.e6.

3. Marcenes W, Kassebaum NJ, Bernabe E, et al. Global burden of oral conditions in 1990-2010: a systematic analysis. J Dent Res. 2013;92(7):592-597.

4. Kassebaum NJ, Baernabe E, Dahiya M, Bhandari B, Murray CJ, Marcenes W. Global burden of severe periodontitis in 19902010: a systematic review and meta-regression. J Dent Res. 2014;93(11):1045-1053.

5. Centers for Disease Control and Prevention (CDC). National diabetes statistics report, 2017. Atlanta, GA: U.S. Department of Health and Human Services 2017. Https://www.Cdc.Gov/diabetes/pdfs/ data/statistics/national-diabetes-statistics-report.Pdf, Accessed August 22, 2019. 
6. International Diabetes Federation (IDF). IDF Diabetes Atlas, 8th ed. Brussels, Belgium: International Diabetes Federation; 2017:150. www.diabetesatlas.org. Accessed August 22, 2019.

7. American Diabetes Association. 2. Classification and diagnosis of diabetes: standards of medical care in diabetes-2019. Diabetes Care. 2019;42(Suppl 1):S13-S28.

8. American Diabetes Association. Standards of medical care in diabetes-2019. Diabetes Care. 2019;42(Suppl 1):S187-S193.

9. Song C, Lyu Y, Li C, et al. Long-term risk of diabetes in women at varying durations after gestational diabetes: a systematic review and meta-analysis with more than 2 million women. Obes Rev. 2018;19(3):421-429.

10. Emerging Risk Factors Collaboration, Sarwar N, Gao P, et al. Diabetes mellitus, fasting blood glucose concentration, and risk of vascular disease: a collaborative meta-analysis of 102 prospective studies. Lancet. 2010;375(9733):2215-2222.

11. Borgnakke WS, Genco RJ, Eke PI, Taylor GW. Ch. 31. Oral health and diabetes. In: Cowie BC, ed. Diabetes in America, 3rd ed. National Institutes of Health/National Institute of Diabetes and Digestive and Kidney Diseases (NIH/NIDDK); 2017. https://www.niddk.nih. gov/about-niddk/strategic-plans-reports/Pages/diabetes-america3rd-edition.aspx. Accessed August 22, 2019.

12. Eke PI, Page RC, Wei E, Thornton-Evans G, Genco RJ. Update of the case definitions for population-based surveillance of periodontitis. J Periodontol. 2012;83(12):1449-1454.

13. Cianciola LJ, Park BH, Bruck E, Mosovich L, Genco RJ. Prevalence of periodontal disease in insulin dependent diabetes mellitus (juvenile diabetes). J Am Dent Assoc. 1982;104(5):653-660.

14. .Demmer RT, Holtfreter B, Desvarieux M, et al. The influence of type 1 and type 2 diabetes on periodontal disease progression: prospective results from the Study of Health in Pomerania (SHIP). Diabetes Care. 2012;35(10):2036-2042.

15. Silvestre F-J, Miralles L, Liambes F, Bautista D, Sola-Izquierdo E, Hernandez-Mijares A. Type 1 diabetes mellitus and periodontal disease: relationship to different clinical variables. Med Oral Patol Oral Cir Bucal. 2009;14(4):E175-E179.

16. Novak KF, Taylor GW, Dawson DR, Ferguson JE 2nd, Novak MJ. Periodontitis and gestational diabetes mellitus: exploring the link in NHANES III. J Public Health Dent. 2006;66(3):163-168.
17. Kumar A, Sharma DS, Verma M. et al. Association between periodontal disease and gestational diabetes mellitus - a prospective cohort study. J Clin Periodontol. 2018;45(8):920-.931

18. Nelson RG, Shlossman M, Budding LM, et al. Periodontal disease and NIDDM in Pima Indians. Diabetes Care. 1990;13(8):836-840.

19. Costa FO, Cota LOM, Lages EJP, et al. Progression of periodontitis and tooth loss associated with glycemic control in individuals undergoing periodontal maintenance therapy: a 5-year follow-up study. J Periodontol. 2013;84(5):595-605.

20. Genco RJ, Genco Doherty F. Common risk factors in the management of periodontal and associated systemic diseases: the dental setting and interprofessional collaboration. J Evid Base Dent Pract. 2014;14(Suppl):4-16

21. Borgnakke WS. "Non-modifiable" risk factors for periodontitis and diabetes. Curr Oral Health Rep. 2016;3(3):270-281.

22. Borgnakke WS. Modifiable risk factors for periodontitis and diabetes. Curr Oral Health Rep. 2016;3(3):254-269.

23. Kocher T, König J, Borgnakke WS, Pink C, Meisel P. Periodontal complications of hyperglycemia/diabetes mellitus: epidemiologic complexity and clinical challenge. Periodontol 2000. 2018;78(1):59-97.

24. Verhulst MJL, Loos BG, Gerdes VEA, Teeuw WJ Evaluating all potential oral complications of diabetes mellitus. Front Endocrinol (Lausanne). 2019;10:56.

25. Monje A, Catena A, Borgnakke WS. Association between diabetes mellitus/hyperglycaemia and peri-implant diseases: systematic review and meta-analysis. J Clin Periodontol. 2017;44(6):636-648.

26. Borgnakke WS, Anderson PF, Shannon C, Jivanescu A. Is there a relationship between oral health and diabetic neuropathy?. Curr Diab Rep. 2015;15(11):93.

27. Guo S, Dipietro LA. Factors affecting wound healing. J Dent Res. 2010;89(3):219-229

How to cite this article: Genco RJ, Borgnakke WS. Diabetes as a potential risk for periodontitis: association studies.

Periodontol 2000. 2020;83:40-45. https://doi.org/10.1111/ prd.12270 